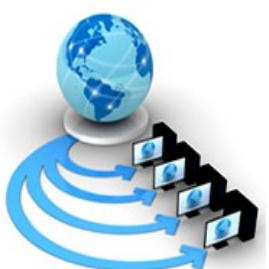

Volume 9, No. 5, September-October 2018

ISSN No. 0976-5697

International Journal of Advanced Research in Computer Science

RESEARCH PAPER

Available Online at www.ijarcs.info

\title{
TECHNIQUE FOR DETECTING SINGLE AND MULTIPLE BLACKHOLE ATTACK ON WIRELESS SENSER NETWORKS
}

\author{
Abhishek soni ${ }^{*}$, Rajneesh Pachouri and Anurag Jain \\ Computer Science\& Engineering, Adina Institute of Science\& Technolog, RGPV, Sagar, India
}

\begin{abstract}
Security is the fundamental issue in wireless sensor networks and attackers are effortlessly altered the Actual behaviour and performance of network. In this research work we give the security plot against single and multiple black hole attack in wireless sensor networks. in black hole attack malicious node behave like a normal node and show that they are part of our network but when the data packets arrives they drops all the packets and reply false value to other nodes. in this proposed scheme we have identified the attackers node that caputers the data packets and nor forwareded to the destination. in wireless sensor network the malicious nodes are only nodes which are not farwareded data packects to goal node. in our proposed scheme we not only detect single black hole but also capable to detect multiple blackhole attack. black hole attack is veryharmful attack, the proposed method is surely identify the malicious node from the dynamic network and increase the network efficiancy.
\end{abstract}

Keywords: Blackhole, WSN, Routing, Security, IDS, Malicious nodes

\section{INTRODUCTION}

Remote Sensor Networks is a self-governing, self arranging system. This system can be sent anyplace easily without no help on any settled framework. There is framework less and brought together organization in this kind of systems. Hubs are consistent from first to last remote interface. The dynamic idea of such kind of systems makes it exceedingly hung to different connection assaults. The basic prerequisites for an anchored remote systems administration are secure conventions which guarantee the tact, accessibility, legitimacy, truth of system. Many existing wellbeing answers for wire arranged systems are inefficacious and wasteful for Mobile impromptu systems (Wireless Sensor Networks) condition. A specially appointed system is the co-agent condition of an arrangement of portable hubs which does not required a hindrance of any brought together framework. A specially appointed system is the briefly settled and made system, which is overseen and worked by taking part hubs. Portable specially appointed system (Wireless Sensor Networks) is a gathering or set of versatile hubs which can contact to each other by utilizing multi-jump remote connections. Versatile specially appointed system does not require any concentrated administration framework and settled system topology of hubs.

Portable specially appointed system is unconstrained, foundation or topology less and self composed system. Remote Sensor Networks has wide territory utilize in light of their self foundation, self creation, and self upkeep. Versatile specially appointed system (Wireless Sensor Networks) is a vital part for correspondence for portable framework. Portable framework or hubs or gadget in the versatile specially appointed system has a flexibility for passage or exit from the system. In a blackhole assault $[1,2]$ an assailant gets parcels from the sender and answer through bogus data of goal., and said in figure 1.2. The An is aggressor hub and $\mathrm{S}$ is sender and $\mathrm{D}$ is collector.

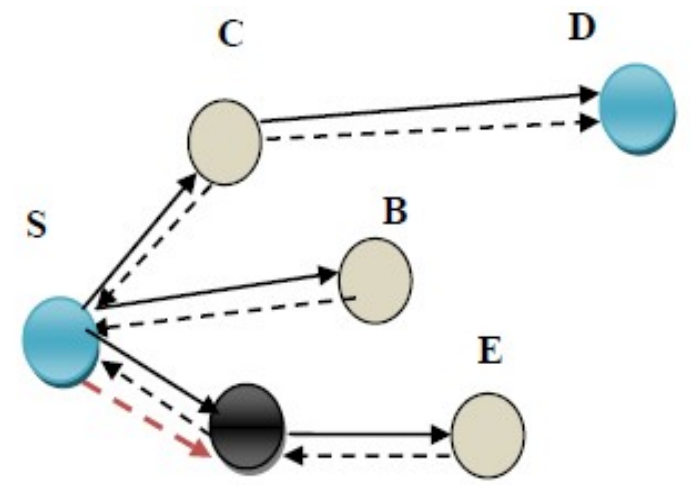

fig:1- Black Hole Attack in Wireless Sensor Network

Versatility mirrors the every now and again change of system topology. Portable hubs in the versatile specially appointed system which has a similar correspondence run are said to be the neighboring hubs and neighboring hubs can contact straightforwardly to each other. Portable hubs in Wireless Sensor Networks can convey to each other by passing the information and control parcels starting with one hub then onto the next hub, which are in a similar remote range. Trusted and co-agent conduct of versatile hubs helps in the correspondence of portable hubs in the Wireless Sensor Networks. The portable hubs in a Wireless Sensor Networks might be workstation, switch, mobile phone, individual advanced aides and so on. Portable Nodes sets up the virtual gathering of association which serves to each other in passing data and control bundles to each other.

The aggressor in arrange is existing in remote transmission scope of a solitary bounce, it is basic or might be conceivable different and drop every one of the bundles touch base with 
preferable metric over a typical multihop course. The blackhole assault is the steering assault and their conduct is likewise similar to as unique blackhole implies catch every one of the information bundles. It is additionally workable for the assailant to forward each piece over the blackhole straightforwardly. Because of the idea of remote transmission, the assailant can make a blackhole notwithstanding for bundles not routed to itself by that all parcels are sent through aggressor and genuine goal sit tight for information. In world, such an unselfish hubs is ordinarily remarkably troublesome to acknowledge and after that we consistently see malevolent hubs conjointly commitment inside a similar system. Some of these are aggressor hubs that influence the whole task of system.

\section{RELATED WORK}

The past work in field of blackhole is specified in this area. These work are additionally proficient and gives data about the work is as of now done in field of assault.

In [3] Sathish $M$ et.al proposed security plan to shield the system from dark opening assaults, it is vital to find pernicious hubs amid the course disclosure process, when they pass created RREP copying the source hub. The proposed technique does definitely the same. In view of next jump data and goal arrangement number that can be extricated from RREPs, this plan handles single and collective dark opening assaults with mitigated computational, directing and capacity overhead.

In this work [4] V. Keerthika et.al proposed Direct/aberrant trust is processed utilizing standardized Route Reply bad conduct factor, interface quality, and effective conveyances to moderate dark gap assault. The speculation that hub ability is likewise fundamental for productive working of the system isn't considered. In this work it is proposed to incorporate system parameters to process trust. Hubs travel a long separation in space among one in WIRELESS SENSOR NETWORKSs and are not particular of another's dependability in light of not gathering adequate confirmation. The model is expected to speak to vulnerability in like manner with regular vulnerability.

In this paper [5] Raquel Lacuesta et.al can build up a protected self-designed condition for information dissemination and assets and administrations sharing among clients. A customer is fit to associate the system since he/she knows some individual to encourage has a place with it. In this way the legitimate or ensured expert is dispersed between the addicts that trust the new someone who is addicted. The system administration is likewise disseminated, which enables the system to have an appropriated name benefit. We apply topsyturvy cryptography, where every gadget has an open private key combine for gadget distinguishing proof and symmetric cryptography to trade session keys between hubs. There are no unknown clients, since classification and legitimacy depend on client distinguishing proof. Unconstrained specially appointed systems require all around characterized, effective, and easy to understand security instruments.
In [6] Raj et al. proposed DPRAODV an extra check is done to discover whether the RREP $\mathrm{se}<\mathrm{L}$ no esteem is higher than the limit an incentive when contrasted with typical AODV. On the off chance that the RREP $\mathrm{se}<\mathrm{L}$ no esteem is higher than the edge esteem, the hub is thought to be vindictive and that hub is added to the boycott. As the hub recognizes a pernicious hub, it sends an ALARM parcel to its neighbors. This ALARM bundle has boycotted hub as a parameter. Afterward, if any of the other hub/s gets the RREP bundle it guarantees the boycott condition. In the event that that hub or hubs are boycotted, it essentially disregards it and does not get answer from that hub once more.

In [7] Panthi N.K et. al. had proposed a plan which substantiate the security of information as well as ensures the unremitting activity of moderator by using a fake operator and composite affirmation method. The system reproduction additionally represent that no specialist barren for a couple of number of noxious hubs. A few shortcomings affirm the expansion in delay, they have not thought about the security of observing specialist, and the handling time required is additionally higher. They overview three methodologies for the problem of versatile specialist assurance. The three security approaches are favored in light of the fact that every one is remarkably executed and has qualities that different methodologies don't need to anchor arrange. They pick fractional outcome validation code approach since it can shield results from portable specialists. Processing with scrambled capacities approaches is chosen since it attempts to clutter code and information together. A muddled framework approach is picked in light of the fact that it scrambles an operator's code so that nobody can pick up an entire comprehension of its capacity.

In [8], L.Tamilselvan et al., proposes the idea of 'Loyalty Table. Here, each taking part hub is dispensed a specific devotion level, a proportion of dependability. At whatever point a sender hub in organize is communicates a RREQ and hold up, the got RREPs are assemble in its Response Table. In the event that the normal of the devotion level of RREP sending hub (RREPN) and its next jump hub (NHN) in the course is observed to be over a foreordained edge, the RREPN is considered as reliable. Thusly, on the receipt of various RREPs, the one with the most noteworthy loyalty level is chosen. Be that as it may, if numerous hubs have a similar constancy level, the RREP with the insignificant bounce check is picked. At last, directing is expert by means of the chose way.

In [9] Sun B proposed conspire in light of the arrangement of coordinated associated or neighbor hubs data, a strategy is intended to anchor organize from the dark gap assault, which comprises of two sections: discovery and reaction. In discovery method, two noteworthy advances are: initial step gather neighbor set data. Second step decide if there exists a dark opening assault. In answer methodology of demand, Sender hubs in arrange are sends a Modify Route Entry (MRE) control parcel to the Destination hub to shape a right lonk by changing the steering passages of the transitional hubs (1M) from source to goal. This plan adequately and proficiently distinguishes dark 
gap assault without acquainting much steering control overhead with the system.

In this paper [10] NPV plot is good with best in class security models, In this point of view, the test is to perform, without confided in hubs, a completely circulated, lightweight NPV technique that empowers every hub to procure the areas promoted by its neighbors, and survey their honesty. They propose a NPV convention that has the accompanying highlights:

- It is thought for WIRELESS SENSOR NETWORKS dynamic or capricious conditions, and all things considered, it doesn't depend on the nearness of a confided in foundation or of from the earlier reliable hubs.

- It use participation yet enable hubs to play out all substantiation occasions self-sufficiently. This plan has no requirement for broadened associations, e.g., to achieve an amicability among different hubs, making this plan appropriate for both low and high portability situations.

- It is responsive, implying that it very well may be executed by any hub, anytime, without previous attention to the area.

- It is energetic other than autonomous and conspiring foes. It is unimportant, as it produces low overhead movement.

In this paper [11] Zhang et al. proposed a blackhole location plot in light of succession number checking of the RREP bundles. They considered a situation where a halfway hub is an aggressor and recommended that, at whatever point a hub sends a RREP back to a source hub, the middle of the road hub ought to likewise produce a demand for an arrangement number to the goal hub. The goal hub reacts by sending a parcel containing its arrangement number to the source hub. The source hub at that point checks the freshness of the course by contrasting the succession number of the RREP got from the middle of the road hub (suspect) with the arrangement number answer parcel from the goal hub; it subsequently identifies an assault if the correlation comes up short. Be that as it may, the presentation of two new parcels with each answer builds the directing overhead as well as the hubs need to guarantee that the assailant does not drop or alter these succession demand and grouping answer messages.

In this work [12] Panagiotis Papadimitratos and Zygmunt J. Haas is principally ponder the increase of course demand and answer parcels and inside the spin-off each message kind is spoken to severally. Be that as it may, it's feasible for SRP to control in an exceedingly a great deal of general setting, where, for instance, a course answer is affixed to a data parcel. Amid this work a course revelation convention that mitigates the hurtful impacts of such malignant conduct, on give adjust availability data. Their convention ensures that imagined traded off or replayed course answers would either be rejected or ne'er reach back the questioning hub. In addition, the convention responsiveness is protected underneath varying kinds of assaults that endeavor the directing convention itself. The main request of the proposed topic is that the presence of a security relationship between the hub starting the inquiry and along these lines the looked for after goal. In particular, no presumption is shaped concerning the middle of the road hubs, which can show incautious and malevolent conduct. The wide acknowledged strategy inside the Wireless Sensor Network setting of course revelation in view of broadcasting inquiry bundles is that the premise of our convention. A considerable measure of particularly, as question parcels navigate the system, the handing-off middle of the road hubs add their image (e.g., ip address) in the inquiry bundle header. when one or a ton of inquiries gain the needed goal, answers that contain the amassed courses are returned to the questioning hub; the source at that point may utilize at least one of these courses to forward its data. In this exploration work [13] K. S. Sujatha, Vydeki Dharmar, R. S. Bhuvaneswaran, proposed Design of Genetic Algorithm based IDS for Wireless Sensor Networks. In this work proposed a technique to investigate the disclosure to assaults in AODV steering convention, especially the most well-known system layer assault, Blackhole assault and to build up a detail based Intrusion Detection System (IDS) utilizing Genetic Algorithm approach. The proposed framework depends on Genetic Algorithm, which breaks down the practices of each hub and gives insights about the assault. Hereditary Algorithm Control (GAC) is an arrangement of different guidelines in light of the indispensable highlights of AODV, for example, Request Forwarding Rate, Reply Receive Rate et cetera.

In this exploration work [14] Dr Karim KONATE, GAYE Abdourahime, proposed an Attacks Analysis in portable specially appointed systems: Modeling and Simulation. In this title display work is committed to examine assaults and countermeasures in Wireless Sensor Networks. After a short prologue to what Wireless Sensor Networks are and organize security we exhibit an overview of different assaults in Wireless Sensor Networks relating to fall flat directing conventions. We likewise exhibit the diverse devices utilized by these assaults and the instruments utilized by the anchored steering conventions to counter them. In this characterized the idea of DoS like its different kinds. They displayed a few options of DoS assaults met in Wireless Sensor Networks, their working procedure accordingly the systems utilized and the conventions which execute them to counter these assaults.

In this paper [15] N. Gandhewar, R.Patel, proposed Detection and Prevention of Sinkhole Attack on AODV Protocol in Mobile Adhoc Network. This work chiefly centers around sinkhole issue, its outcomes and presents component for discovery and avoidance of it on the setting of AODV convention. Sinkhole is one of serious sort of assault which endeavors to pull in a large portion of system movement towards it and corrupt the execution of system. AODV directing convention is chiefly examined under wormhole and blakhole, and flooding assault, which needs to break down under different sorts of assault moreover. It additionally indicates execution of AODV with no sinkhole assault, under assault and in the wake of applying our instrument as recreation result got for certain variety of hubs in 
arrange, by considering execution measurements as throughput, PDR, End to end delay and Packet misfortune.

In this paper [16] P.K Singh, G. Sharma, proposed An Efficient Prevention of Black Hole Problem in AODV Routing Protocol in Wireless Sensor Networks. In this work an answer for the dark opening assault in one of the notable directing calculation, Ad-hoc on request remove vector (AODV) steering, for the Wireless Sensor Networks. The dark opening assault is one of such security dangers. In this assault, a malignant hub dishonestly publicize most brief way to the goal hub with an intension to upset the correspondence. The proposed technique utilizes unbridled mode to distinguish malevolent hub (dark gap) and proliferates the data of vindictive hub to the various hubs in the system.

In this paper [17] Jian-Ming Chang, Po-Chun Tsou, Han-Chieh Chao, Jiann-Liang Chen, anticipated CBDS: A Cooperative Bait Detection subject is stop pernicious exercises of aggressor hubs for Wireless Sensor Networks upheld half and half guard outline. They offered an instrument to find vindictive hubs propelling blackhole or grayhole assaults and helpful area assaults, known as Cooperative Bait Detection topic (CBDS). It incorporates the proactive and responsive guard designs, and at irregular participates with an arbitrary neighboring hub. By exploitation the address of the nearby hub in light of the fact that the trap goal address, it lures vindictive hubs to answer RREP and recognizes the pernicious hubs by the proposed switch following system and subsequently keeps their assaults.

\section{Methodology}

The assailant nearness in arrange is certain performing pernicious exercises in unique system. In Wireless Sensor Networks assailant hubs are easily enter in arrange and respond as an ordinary hub. The malignant working is occurring at the season of correspondence. The assailant or aggressors are being the piece of system and this aggressor nearness is debases the system execution. In this examination the single blackhole identification and various blackhole location strategy is proposed. The proposed conspire is distinguished the aggressor and furthermore assailants in unique system through malevolent profile of aggressor. The aggressor profile is not quite the same as the other ordinary hubs profile and furthermore the assailant conduct is to not forward the information parcels to real goal. The aggressor is drop these information parcels in arrange. The conduct of aggressor is distinguished through making the steering blunder. This bungle passage is available in the assailant profile. The proposed calculation is demonstrates the identification and avoidance of single blackhole assault and different blackhole assault independently in arrange. The preventer hub can perceived the assailant malevolent exercises in arrange. The disease in the system is tallied by preventer hubs on the grounds that these hubs are really affirming the assailant nearness.

Algorithm: Single Blackhole node detection and prevention Input:

M: mobile nodes

I: intermediate nodes
B: blackhole node

P: preventer node

S: Source node

D: destination node

rp: : routing packet

ack: acknowledge

Seq: higher sequence number

AODV: routing protocol

$\lambda$ : radio zone $550 \mathrm{~m}$

Output: blackhole node detection, percentage of infection, PDR, NRL, throughput

Procedure:

Step1: S execute (AODV)

Step2: Generate AODV packet (S, D, $\lambda, \mathrm{rp}$ )

Step3: If $I$ in $\lambda \& \&$ I != D Then

If $(\mathrm{I}==\mathrm{B})$ then

I generate Seq

Send (ack, Seq, S)

$\mathrm{S}$ established route

Send packet(S, I, data)

Else

I generate route table

Forward route packet to next-hop

Increase count

\section{End if}

Else If $\mathrm{I}$ in $\lambda \& \& \mathrm{I}==\mathrm{D}$ Then

$D$ receives route packet

Create reverse route table

Generate ack packet

Send (ack, D, S)

Send packet(S, I, data)

Else

$\mathrm{D}$ unreachable or $\mathrm{D}$ not in range

End if

\#Blackhole node detection \& prevention

Step4: P watch activity of I node

If I generate Seq \& send ack to $S$ Then

I <-suspicious as B node

$\mathrm{P}$ watch the activity of I node

If I drop data by self loop then

I confirm B node

Block I node

$\mathrm{P}$ generate packet \& broadcast to all neighbour

$S$ receives packet

Re-execute route without participation of B node

Send packet(S, I, data)

\section{End if}

\section{End if}

The same procedure with multiple preventer nodes is applied on multiple attackers. These attackers are very harmful because they are covering the whole network area and due to that the drooping and infection of attacker is more in network. The preventer nodes quantity is decided on the basis of cover all the malicious nodes in network. If the node density is high then in that case it is necessary to enhance the quantity preventer nodes also.

Algorithm: Multiple Blackhole nodes detection and prevention Input:

B: b1, b2 ....bi....... bn (blackhole nodes) 
P: p1, p2 ....pi. pn (preventer nodes)

rp: : routing packet

ack: acknowledge

Seq: higher sequence number

utput: blackhole node detections, percentage of infection, PDR, NRL, throughput

If bi \& bj route then Capture all source node by different Seq to $\mathrm{Si} \& \mathrm{Sj}$ node respectively

Send ((acki, Seqi, Si),( ackj, Seqj, Sj)

Si \& Sj established different route

All path are infected by B behaviour

End if

\#Blackhole nodes detection \& prevention

Step4: $\mathrm{Pi}, \mathrm{Pj}$ watch activity of Ii , Ij node

If Ii generate Seqi \& Ij generate Seqj Then

$\mathrm{Ii}, \mathrm{Ij} \leftarrow$ suspicious as B node

$\mathrm{Pi} \& \mathrm{Pj}$ watch the activity of $\mathrm{Ii}, \mathrm{Ij}$ in separately

If $\mathrm{Ii}, \mathrm{Ij}$ drop data by self loop then

Ii, Ij confirm B node

Block Ii, Ij node

$\mathrm{Pi}, \mathrm{Pj}$ generate packet $\&$ broadcast to all neighbour

$\mathrm{S}$ receives packet

Re-execute route without participation of B node

Send packet(S, I, data)

End if

The proposed security scheme is not detect the attacker but also this scheme is convey information of attacker to all nearby nodes. The preventer nodes are work for it to convey attacker information in network by that sender as well as intermediate nodes are not participating in routing or forward request to sender where attacker is exist. The preventer nodes are also block the attacker malicious functioning and provide the secure communication.

Consider multiple preventer nodes for multiple blackhole attacker.

\section{RESULTS AND DISCUSSION}

\section{UDP End Data Receiving Analysis}

The bundles accepting in nearness of blackhole assault and in nearness of IDS is specified in given diagram. The execution of better bundles accepting is gives the more advantageous system execution. In this chart the execution of parcel getting in nearness of blackhole assault is relatively immaterial in organize yet in the wake of applying IDS the execution in arrange is enhances and furthermore the bundle misfortune is limited. The nearness of aggressor hubs in arrange is extremely exceptionally destructive for appropriate correspondence in organize.

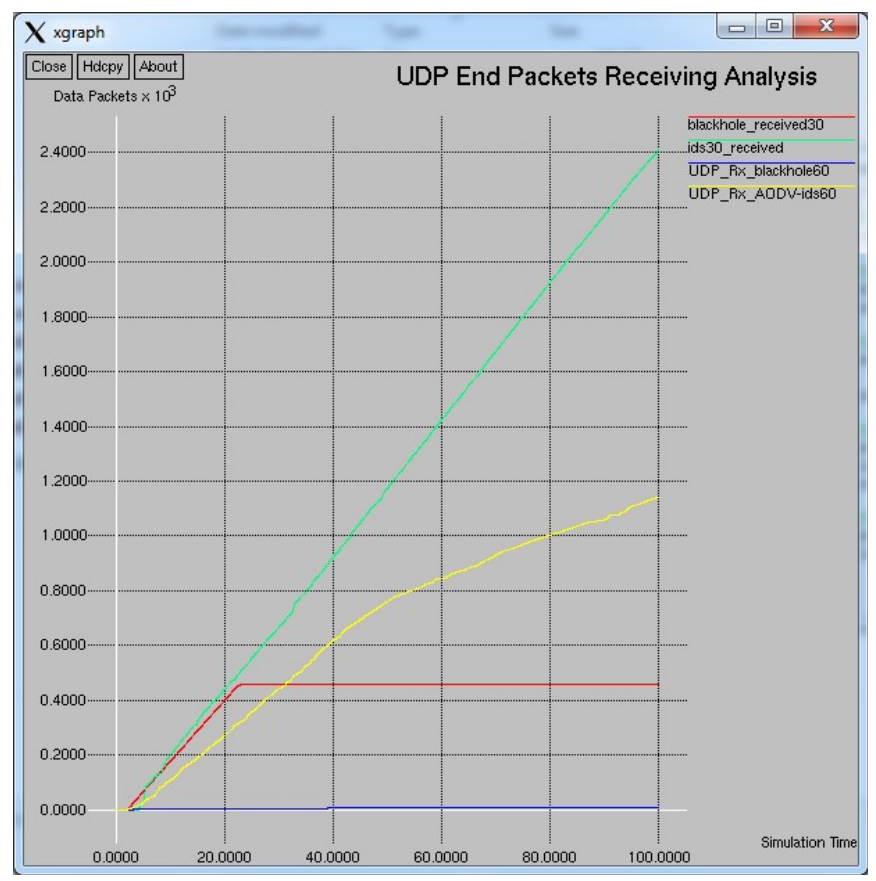

Fig.3:- UDP End Data Receiving Analysis

2. UDP End Packet Loss Analysis in Node Density 30 and 60

The quantity of hubs in Wireless Sensor Networks is likewise demonstrates the likelihood of more load in organize. In this chart the execution of UDP (User Datagram Protocol) bundles getting is estimated and see that the parcels accepting in both the situation of hub thickness 30 and 60 . In this chart the bundle misfortune in 30 hub situation in nearness of blackhole is about just 340 parcels and in nearness of 60 hub situation is about unimportant packts are misfortune yet in addition parcel accepting is poor. In the wake of applying IDS security the execution of system are gives better execution and furthermore hinder the aggressor vindictive exercises.

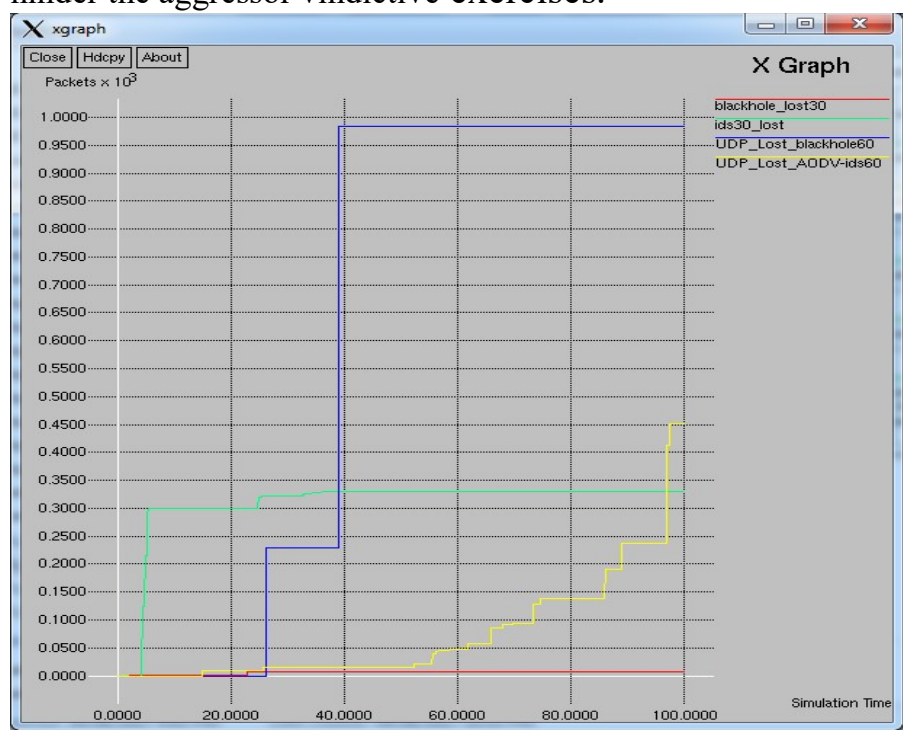

Fig.4 UDP End Loss Analysis 


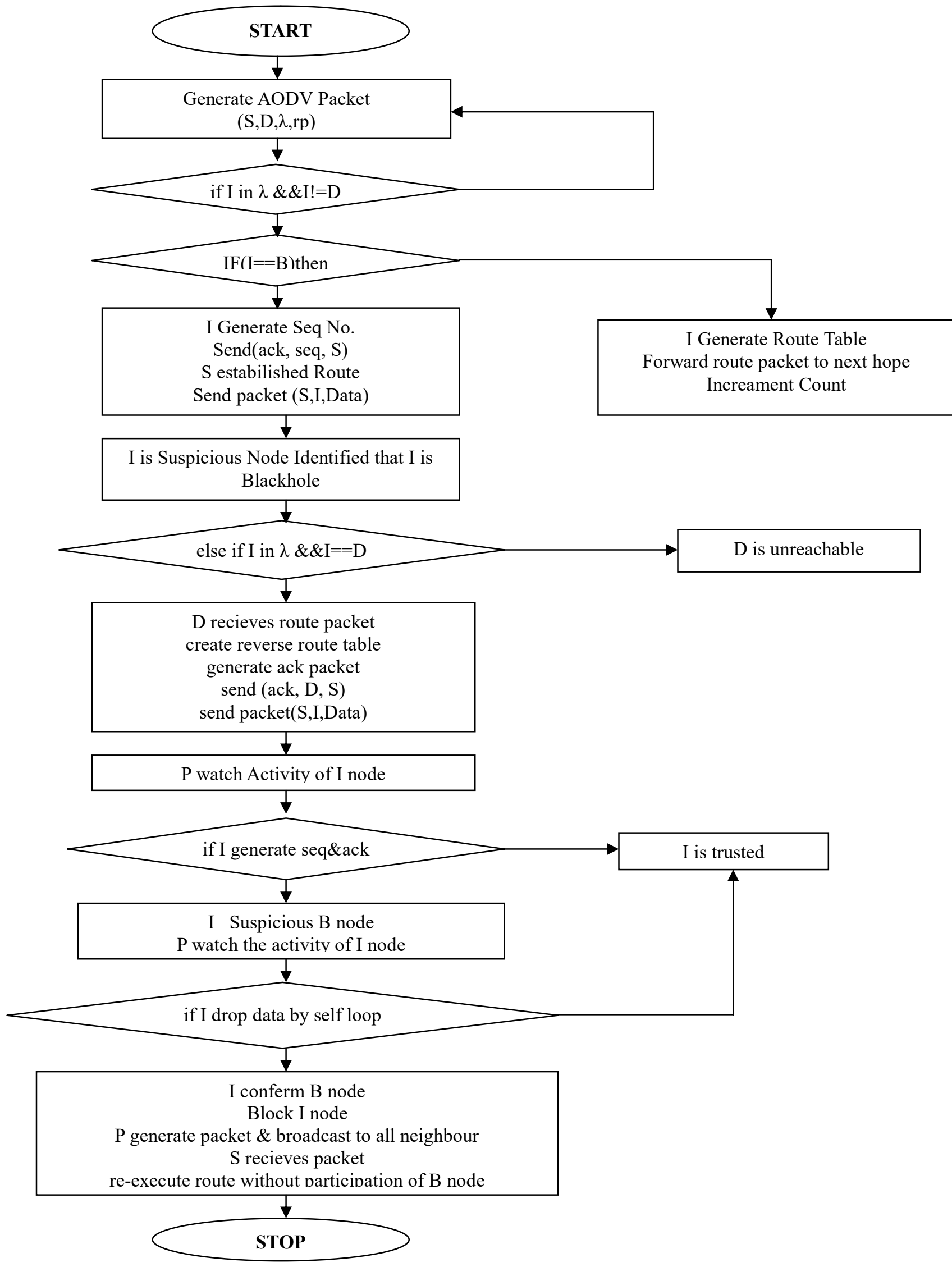

fig 2:- flowchart for proposed Algorithm 


\section{Attacker TCP End Data Receiving Analysis}

In TCP (Transmission Control Protocol) the quantity of sender are send information in organize and furthermore get Acknowledgement(ACK) from recipient of fruitful information conveyance. In the event that the ACK isn't gotten by sender of fruitful information conveyance then it implies information is drop because of some specific reason in arrange. In this diagram the main TCP end bundles getting examination is assessed in nearness of aggressor. The quantity of TCP associations in two unique situations of 30 hubs and 60 hubs. The TCP getting clog window greatest size is about just 12 parcels of TCP 1 association at time around 30 seconds. In this chart rest of the associations are indicates just a single or two bundles in organize because of essence of aggressor vindictive conduct

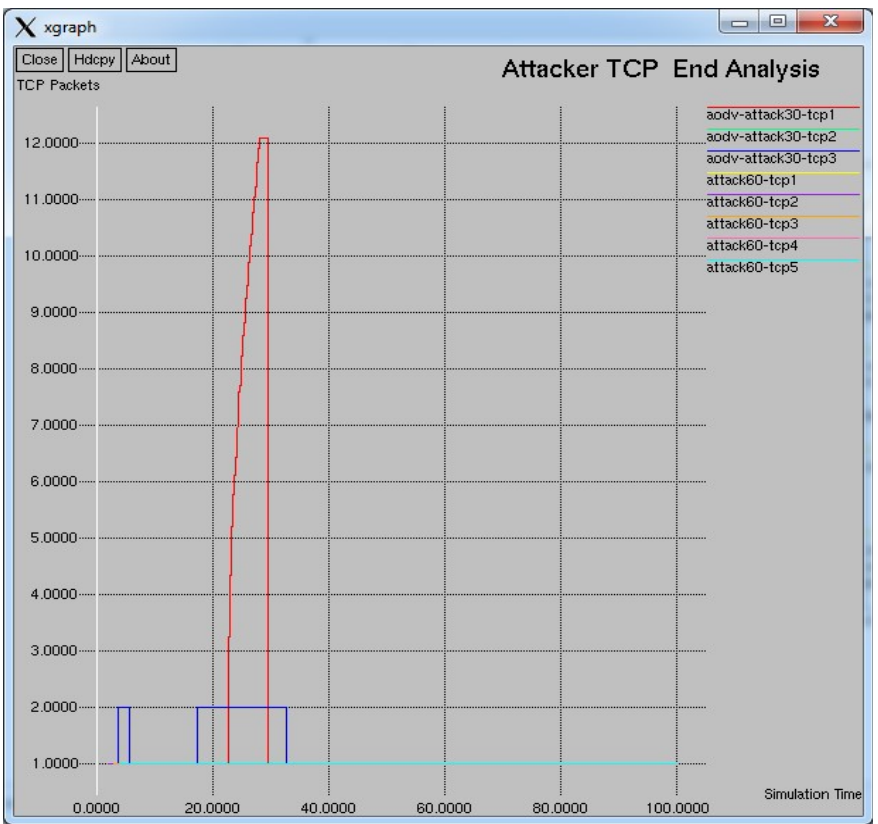

Fig.5 Attacker TCP End Receiving Analysis

\section{IDS TCP END DATA RECEIVING ANALYSIS}

The information dropping probability in the event of TCP is relatively unimportant on the grounds that from ACK we recognized the dropping status of bundles which are dropped because of any reason in organize. In this diagram the getting TCP clog window investigation in nearness of IDS is assessed and sees that the execution of proposed security conspire is giving better outcomes. Here the bundle accepting is gives the better outcomes and size of clog window is comes to up to 70 parcels. The execution of all blockage window of situation 30 and 60 is giving the attractive outcomes in arrange. The proposed IDS is extremely viable because of totally incapacitate aggressor contamination in organize.

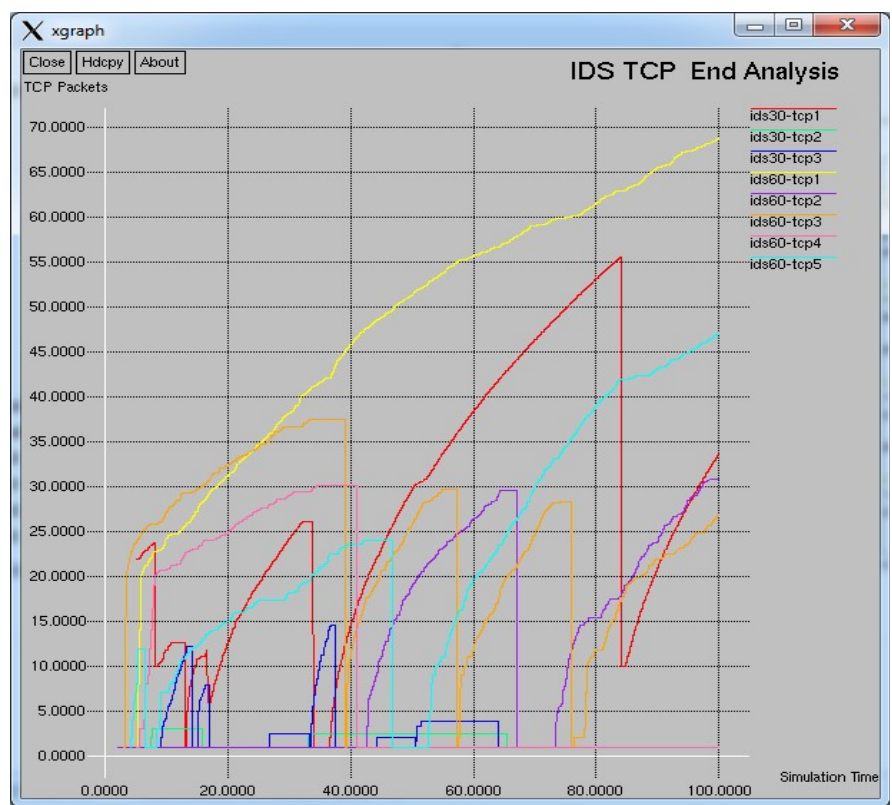

Fig.6 Attacker TCP End Receiving Analysis

\section{ATTACKER INFECTION ANALYSIS}

The assailant nearness in organize is unquestionably demonstrates the debasement in arrange execution. In this chart the misfortune rate examination of single blackhole assailant and various blackhole aggressors is measure. The misfortune rate is the measure of information is dropped by aggressor in organize. Here the loss of information because of aggressor is tallied in nearness of various blackhole nearness and in addition contrast with single blackhole nearness. In nearness of various aggressors misfortune rate is comes to up to $48 \%$ and stop at $35 \%$ in reenactment time of 100 seconds. The proposed IDS evacuates the contamination of assailant or aggressors in organize and gives the more advantageous correspondence in the middle of sender and recipient in unique system.

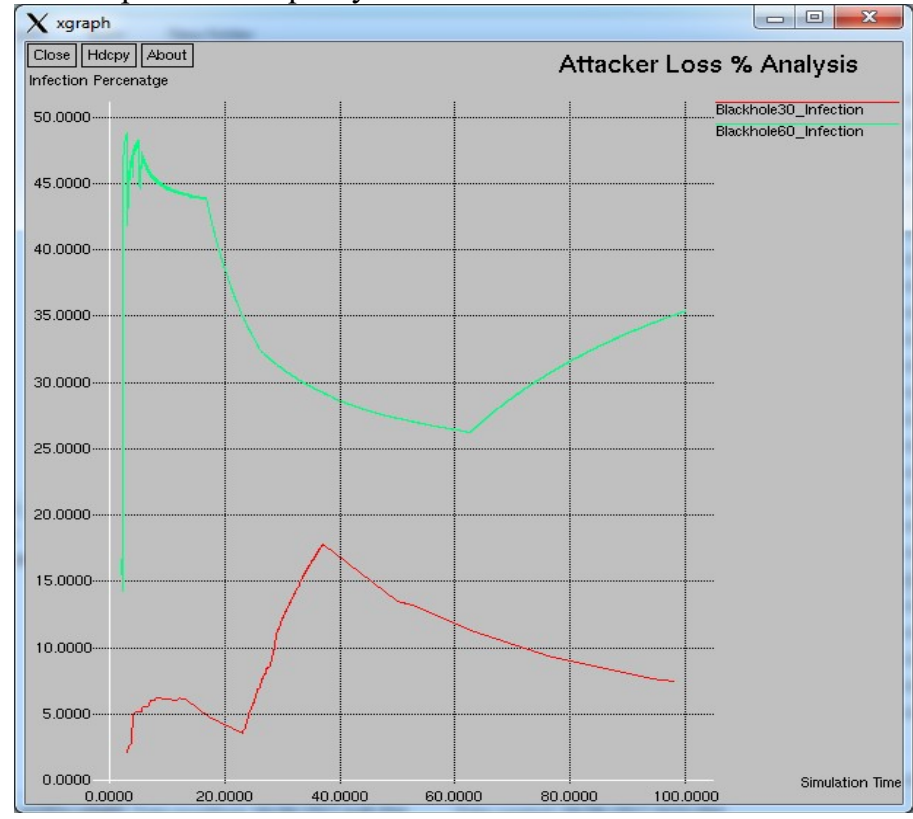

Fig.7 Attacker Loss Analysis 


\section{Throughidt Performance Analysis}

The execution of system is assessed in per unit of time is called as throughput. In throughput fundamentally the tallying of bits are considered for estimating system execution. In this diagram execution of assault and IDS is assessed the execution based on number of parcels got at goal in every second. In this chart the throughput execution of single blackhole and various blackhole assault is demonstrates immaterial bundles getting in organize. In nearness of single blackhole the execution is included up to 75 seconds organize yet in various truly execution is unimportant. The proposed IDS is enhances throughput execution and gives parcels getting up to 550 bundles and 640 bundles in unit time in powerful system. The proposed security conspire is gives better execution and secure correspondence in powerful system.

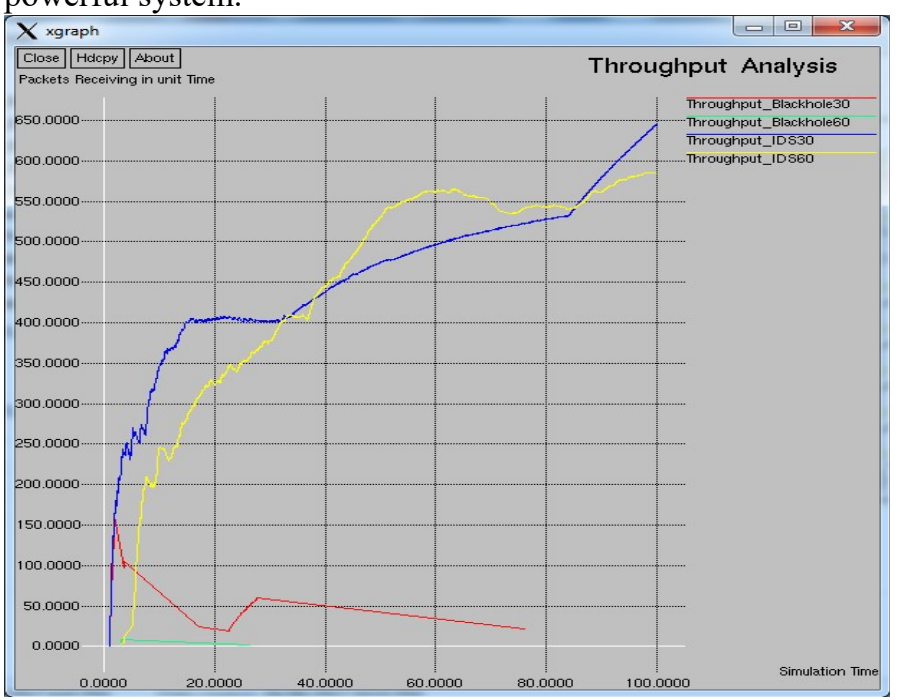

Fig.8 Throughput Analysis

\section{PDR PERFORMANCE ANALYSIS}

The Packet Deliver Ratio (PDR) is really speaks to rate measure of information in arrange in nearness of aggressor and IDS. The quantity of bundles in nearness of various blackhole assault is dropped more in organize as contrast with single blackhole assault in arrange. The PDR \% in nearness of single blackhole assailant is about scopes to $84 \%$ and last estimation of PDR is recorded up to $70 \%$ at time around 30 seconds. After that not a solitary bundle is gotten at goal. If there should arise an occurrence of different blackhole PDR esteem is tallied up to end of reenactment yet insignificant. The proposed IDS is

obstructed the malevolent exercises in organize and gives secure directing execution in powerful system. In both the cases IDS is viable and furthermore utilize various IDS hubs in nearness of numerous aggressors is organize.

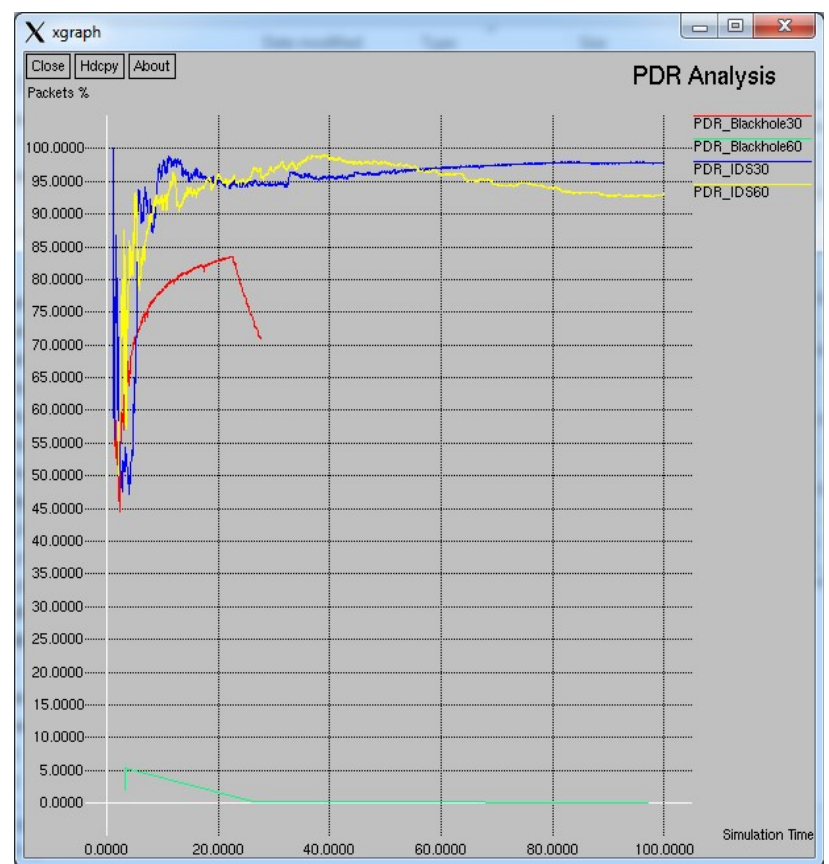

Fig.9 PDR Analysis

\section{BlackHole AtTaCker Drop Analysis}

The aggressor point is just to drop the information parcels in organize. These information bundles are contain the important data of sender. The assailant is middle of the road hub acts like a typical hub in system and this hub nearness is misfortune the enormous number of information parcels in arrange. In the table 1the assailant hub and the loss of parcels because of aggressor is said. The aggressor examination in nearness of single blackhole assailant or different blackhole assailant are gives the real data of information misfortune.

Table 1:- Blackhole Node Identification and Data Loss Analysis

\begin{tabular}{|c|c|}
\hline \multicolumn{2}{|c|}{ Node identification in 30 Nodes Scenario with data loss } \\
\hline Attacker Node & Total Non-Authentic Packets \\
\hline 9 & 380 \\
\hline \multicolumn{2}{|c|}{} \\
\hline Node identification in 60 Nodes Scenario with data loss \\
\hline 13 & 2255 \\
\hline 17 & 6 \\
\hline 29 & 1358 \\
\hline 34 & 758 \\
\hline 39 & 598 \\
\hline
\end{tabular}

Table 2:-summarized performance analysis

\begin{tabular}{|l|c|c|c|c|}
\hline Metrics & $\begin{array}{c}\text { performance } \\
\text { in 30 nodes } \\
\text { scenario }\end{array}$ & $\begin{array}{c}\text { performance } \\
\text { in 30 nodes } \\
\text { scenario }\end{array}$ & $\begin{array}{c}\text { performance } \\
\text { in 30 nodes } \\
\text { scenario }\end{array}$ & $\begin{array}{c}\text { performance } \\
\text { in 30 nodes } \\
\text { scenario }\end{array}$ \\
\hline send & 2609 & 7034 & 5477 & 8218 \\
\hline receive & 551 & 7 & 5352 & 7643 \\
\hline $\begin{array}{l}\text { packet } \\
\text { capture }\end{array}$ & 380 & 4975 & 0 & 0 \\
\hline PDF & 21.12 & 0.1 & 97.72 & 93 \\
\hline NRL & 4.34 & 512.57 & 0.31 & 0.52 \\
\hline
\end{tabular}




\begin{tabular}{|l|c|c|c|c|}
\hline $\begin{array}{l}\text { AVERAGE } \\
\text { e-e } \\
\text { delay(ms) }\end{array}$ & 73.5 & 51.5 & 254.91 & 454.37 \\
\hline $\begin{array}{l}\text { data } \\
\text { packets } \\
\text { dropped }\end{array}$ & 2058 & 7027 & 125 & 575 \\
\hline
\end{tabular}

\section{SUMMARIZED PERFORMANCE ANALYSIS}

The abridged execution of system in nearness of aggressor and IDS is specified in table1. In this table the different execution of two distinct situations of hub 30 (single blackhole) and hub 60 (various blackhole) are assessed up to end of recreation time. In this execution the assailant nearness is plainly speaks to the tremendous loss of information in organize because of bundle catching by aggressor. The postponement in nearness of assailant is least in light of the fact that less measures of parcels are gotten at goal and just the defer estimation is depend based on bundles are gotten at goal.

\section{CONCLUSION AND FUTURE SCOPE}

The single assailant hub nearness is destructive for organize then the numerous blackhole impact is extremely more hurtful for organize. The same malignant capacity is performed by other assailant hubs by that bundle dropping is enhances and entire system are effortlessly secured by aggressors for infusing more contamination. For enhancing system execution, we gives the solid security plot based on bundle dropping conduct of hubs in organize. This exploration is exceptionally helpful in field of security to assess the system execution if there should be an occurrence of assault and IDS. The assault in Wireless Sensor Networks is effortlessly misfortune the information and debases the system directing execution. The past work is gives the thought regarding how the distinctive security conspire is apply the best possible methodology to anchor Wireless Sensor Networks directing execution.

The assailant nearness is misfortune all information of system just a few information is conceivable to convey in goal specifically reproduction time. The proposed IDS is perceived the conduct of blackhole assault by dropping property of aggressor and furthermore their quality is one bounce tally remove from sender. The proposed IDS conduct is keep up consistency for watching system conduct. The quantity of malevolent hubs amount is additionally distinguished by same bundle dropping conduct. The recreation of system is execution in 30 hubs and 60 hubs. In both the situation aggressor impact is extremely horrible however in the wake of applying IDS assailant impact is controlled and additionally obstructed by IDS in arrange. The execution of system is enhances applying proposed security plot that enhances PDR, throughput and limits parcel dropping in arrange. In this plan the location depends on RSS (Received Signal Strength) of versatile hub and if hub is drop bundles then their RSS is week. Presently check the unwavering quality of hub based on parcel dropping. The blackhole aggressor is bundle dropping assailant and different assaults like Tunnel assault is likewise the bundle dropping aggressor in powerful system. In future we proposed the novel security conspire against Tunnel assault. The proposed conspire is likewise connected on burrow assault in Remote Sensor Networks.

\section{REFERENCES}

[1] Horng, Shi-Jinn, et al. "A novel intrusion detection system based on hierarchical clustering and support vector machines." Expert systems with Applications 38.1 (2011): 306-313.

[2] Hayoung Oh," Attack Classification based on Data Mining Technique and its application for Reliable Medical Sensor Communication", International Journal of Computer Science and Applications, Vol.6, No. 3, pp 20 - 32, 2009.

[3] Mohit Malik, Namarta kapoor, Esh naryan, Aman Preet Singh," Rule Based Technique detecting Security attack for Wireless Sensor network using fuzzy logic", International Journal of Advanced Research in Computer Engineering \& Technology,Volume 1, Issue 4, , ISSN: 2278 - 1323, June 2012.

[4] Reda M. Elbasiony, Elsayed A. Sallam , Tarek E. Eltobely ,Mahmoud M. Fahmy ," A hybrid network intrusion detection framework based on random forests and weighted k-means" Ain Shams Engineering Journal”, vol 4, pp.753-762,2013.

[5] Levent Koc , Thomas A. Mazzuchi, Shahram Sarkani,“A network intrusion detection system based on a Hidden Naïve Bayes multiclass classifier”, Elsevier,pp.13492-13500, 2012.

[6] Wenying Fenga, Qinglei Zhangc, Gongzhu Hud, Jimmy Xiangji Huange, "Mining network data for intrusion detection through combining SVMs with ant colony networks", Elsevier, pp. 127140,2013

[7] Megha Bandgar, Komal dhurve, Sneha Jadhav,Vicky Kayastha,Prof. T.J Parvat, " Intrusion Detection System using Hidden Markov Model (HMM)", IOSR Journal of Computer Engineering (IOSRJCE) e-ISSN: 2278-0661, p- ISSN: 22788727Volume 10, Issue 3, pp.66-70, (Mar. - Apr.2013).

[8] Dat Tran, Wanli Ma, and Dharmendra Sharma,"Network Anomaly Detection using Fuzzy Gaussian Mixture Models", International Journal of Future Generation Communication and Networking, pp.37-42, 2012.

[9] Vahid Golmah, “ An Efficient Hybrid Intrusion Detection System based on C5.0 and SVM", International Journal of Database Theory and Application Vol.7, No.2 ,pp.59-70, (2014).

[10] Punam Mulak, Nitin R. Talhar, "Novel Intrusion Detection System Using Hybrid Approach", International Journal of Advanced Research in Computer Science and Software Engineering, Volume 4, Issue 11, ISSN: 2277 128X, November 2014.

[11] Venkata Suneetha Takkellapati1 , G.V.S.N.R.V Prasad," Network Intrusion Detection system based on Feature Selection and Triangle area Support Vector Machine", International Journal of Engineering Trends and Technology-Volume3Issue42012

[12] Vaishali Kosamkar, Sangita S Chaudhari,'Improved Intrusion Detection System using C4.5Decision Tree and Support Vector Machine",International Journal of Computer Science and Information Technologies, Vol. 5 (2) , pp. 1463-1467, 2014

[13] Levent Koc , Thomas A. Mazzuchi, Shahram Sarkani,“A network intrusion detection system based on a Hidden Naïve Bayes multiclass classifier", Elsevier,pp.13492-13500, 2012.

[14] Dr Karim KONATE, GAYE Abdourahime "Attacks Analysis in mobile ad hoc networks: Modeling and Simulation", 2011 Second International Conference on Intelligent Systems, Modelling and Simulation, pp. 367 - 372, 2011.

[15] N. Gandhewar, R.Patel, "Detection and Prevention of Sinkhole Attack on AODV Protocol in Mobile Adhoc Network", Fourth International Conference on Computational Intelligence and Communication Networks (CICN), pp. 714-718, 2012.

[16] P.K Singh, G. Sharma, "An Efficient Prevention of Black Hole Problem in AODV Routing Protocol in MANET", IEEE 11th International Conference on Trust, Security and Privacy in 
Computing and Communications (TrustCom), pp. $902-906$, 2012.

[17] Jian-Ming Chang, Po-Chun Tsou, Han-Chieh Chao, Jiann-Liang Chen, "CBDS: A Cooperative Bait Detection Scheme to prevent malicious node for MANET based on hybrid defense architecture", 2nd International Conference on Wireless Communication, Vehicular Technology, Information Theory and Aerospace \& Electronics Systems Technology (Wireless VITAE), pp. 1-5, 2011. 